\title{
SUNCT syndrome secondary to prolactinoma
}

\section{S Matharu, M J Levy, R T Merry, P J Goadsby}

\begin{abstract}
Short-lasting unilateral neuralgiform headache attacks with conjunctival injection and tearing (SUNCT) syndrome is a rare form of primary headache disorder, although secondary causes, particularly posterior fossa abnormalities, are well known. We report a case of SUNCT syndrome secondary to a prolactinoma. Administration of dopamine agonists led to complete resolution of the SUNCT attacks. This case, together with other similar case reports in the literature, highlight the importance of excluding a diagnosis of pituitary adenoma in all suspected cases of SUNCT syndrome, especially as the headache can precede more classical pituitary symptoms by a considerable period of time. Clinicians managing patients with suspected SUNCT syndrome should elicit a history of symptoms associated with pituitary neoplasms, perform a magnetic resonance imaging scan of the brain and pituitary, and screen for serum hormonal abnormalities.
\end{abstract}

S hort-lasting unilateral neuralgiform headache attacks with conjunctival injection and tearing (SUNCT) syndrome $^{1}$ is a rare form of strictly unilateral headache that occurs in association with cranial autonomic features. The pain is abrupt in onset, of short duration, stabbing or burning in quality, and accompanied by prominent ipsilateral cranial autonomic features, particularly lacrimation and conjunctival injection. ${ }^{2}$ The natural history of the condition is poorly understood. Generally, a clustering pattern is reported, with active phases erratically alternating with remissions. During symptomatic periods, the frequency of attacks varies from less than 1/day to more than 30/h. Functional magnetic resonance imaging (fMRI) in spontaneous attacks has demonstrated activation of the ipsilateral hypothalamic gray. ${ }^{3}$

Classification criteria for SUNCT syndrome have been proposed that require at least 30 attacks of unilateral, moderately severe, orbital or temporal, stabbing or throbbing pain, lasting for 15-120 s, and associated with at least one of the following cranial autonomic features: conjunctival injection, lacrimation, nasal congestion, rhinorrhoea, ptosis and eyelid oedema. ${ }^{4}$ The attacks have to occur at a frequency of 3-100 daily.

Several cases of secondary SUNCT syndrome, particularly due to posterior fossa abnormalities, have been reported. We report a case of SUNCT syndrome secondary to a prolactinoma.

\section{CASE REPORT}

A 37 year old, right handed, married male presented with an 11 year history of headaches in December 1998. There were no precipitants at onset. Initially he had 1-2 headaches per month but there was a gradual increase in the frequency over time. In the early to mid-1990s he had bouts of daily attacks, alternating with remission periods; he had 2-3 remissions annually, with each remission lasting $2-4$ weeks. In the late 1990s, he had no remissions at all. A typical attack was strictly unilateral on the right. It started as a dull ache in the neck or the occiput and then radiated to the parietal region, vertex, forehead and the temple before becoming centred on the retro-orbital region. The pain was very severe or excruciating and had a squeezing or pressing quality. The usual duration of the pain was 20-30 s, although the range was 10-60 s. The pain came on rapidly, was maintained at a plateau phase, and then resolved rapidly. He denied any superimposed spikes or variations in the severity of the pain. He had 1-6 attacks daily at presentation. The attacks were associated with prominent ipsilateral conjunctival injection and lacrimation, and bilateral facial flushing. He denied all other cranial autonomic features, or any nausea, vomiting, photophobia, phonophobia or osmophobia. He felt restless during the pain and would often pace up and down. He denied any aura symptoms. Occasionally he had a dull interictal pain that lasted $10-60 \mathrm{~min}$. The headaches could be triggered consistently by exercise, particularly cycling, and inconsistently by stressful situations. He denied having any other triggers for the headaches. He was unsure whether there was a refractory period after an attack; the shortest interval between two spontaneous attacks was about $3 \mathrm{~min}$. He could abbreviate the attack by coughing. There was no past history of headaches.

The patient had previously tried ibuprofen, aspirin, distalgesic and indomethacin $50 \mathrm{mg}$ three times daily for the headaches without any benefit. At presentation, he was not taking any drugs. In the past medical history, he had had a tonsillectomy in childhood. His sister was diagnosed as having cancer of the thyroid gland at the age of 21 years. There is no family history of headaches. He is a non-smoker and drinks 25-30 units of alcohol per week.

General and a detailed neurological examination were entirely normal. An MRI scan of the brain revealed a pituitary adenoma on the right extending towards the right cavernous sinus, causing displacement of the right internal carotid artery; the pituitary adenoma also extended into the chiasmatic cistern but did not impinge on the optic chiasm (fig 1). Formal visual field testing was normal. Blood tests showed that the serum prolactin level was markedly elevated at $10000 \mathrm{mU} / \mathrm{L}$ (normal range 0-635) and testosterone was at the lower end of the normal range $(10.1 \mathrm{nmol} / \mathrm{L}$; normal range 9-33) with no elevation of luteinizing hormone (LH) or follicle stimulating hormone (FSH), while free thyroxine, thyroid stimulating hormone (TSH), basal cortisol, adrenocorticotropic hormone (ACTH), oestrogen, and growth hormone $(\mathrm{GH})$ levels were normal. A diagnosis of macroprolactinoma was made.

At that point, the patient was re-interviewed to elicit features of pituitary disease. He reported that, in retrospect, there might have been a slight reduction in libido over the

\footnotetext{
Abbreviations: $\mathrm{ACTH}$, adrenocorticotropic hormone; fMRI, functional magnetic resonance imaging; $\mathrm{FSH}$, follicle stimulating hormone; $\mathrm{GH}$, growth hormone; LH, lutenising hormone; SUNCT, short-lasting unilateral neuralgiform headache attacks with conjunctival injection and tearing; TSH, thyroid stimulating hormone
} 


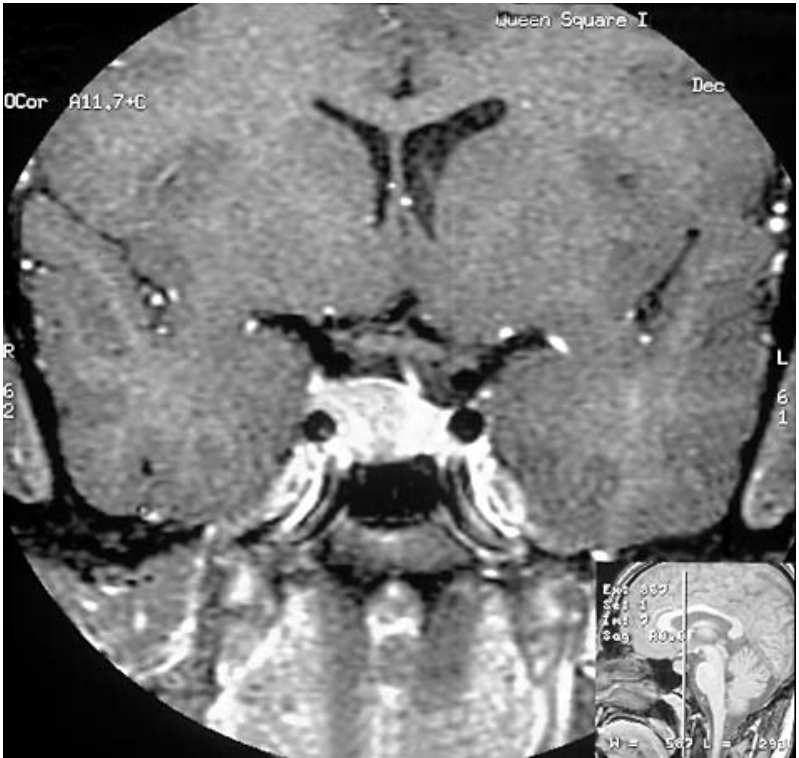

Figure 1 Magnetic resonance imaging scan of the brain, showing a pituitary tumour with right lateral extension invading the right cavernous sinus and causing displacement of the right internal carotid artery.

previous few years, although sexual function had otherwise been normal. He had always had scanty beard growth, but otherwise secondary sexual development and characteristics were normal. There was no galactorrhoea and vision was normal.

The patient was started on bromocriptine $2.5 \mathrm{mg}$ twice daily, and the prolactin level normalised within 3 months. The bromocriptine dose was thereafter reduced to $1 \mathrm{mg}$ twice daily. The headaches started improving shortly after starting bromocriptine and resolved completely within 3 months. A repeat MRI scan of the brain in February 2000 showed that the pituitary adenoma had virtually disappeared. The patient continued taking bromocriptine for 18 months but began to develop nausea and nasal blockage; bromocriptine was therefore substituted with cabergoline $1.5 \mathrm{mg} /$ week. He has been on cabergoline over the last 18 months, on which the prolactin level remains normal and there are no side-effects. He denies having had any further headaches and his libido and energy levels have significantly improved.

\section{DISCUSSION}

The phenotype of this headache satisfies diagnostic criteria for SUNCT. ${ }^{45}$ Medical treatment of the prolactinoma led to a resolution in headache symptoms, which strongly indicates that the prolactinoma was pivotal in triggering the headache, rather than a coincidental pathology.

Most cases of SUNCT syndrome described in the medical literature are primary but several cases of secondary SUNCT have been reported. The secondary SUNCT cases can be divided into two groups: posterior fossa and pituitary abnormalities. There are seven case reports of SUNCT syndrome secondary to a posterior fossa abnormality including: homolateral cerebellopontine angle arteriovenous malformations in two patients; ${ }^{67}$ a brainstem cavernous hemangioma; ${ }^{8}$ a posterior fossa lesion in an HIV/AIDS patient; ${ }^{4}$ severe basilar impression causing pontomedullary compression in a patient with osteogenesis imperfecta; ${ }^{9}$ craniosynostosis resulting in a foreshortened posterior fossa; ${ }^{10}$ and ischaemic brainstem infarction. ${ }^{11}$

There are four case reports of SUNCT syndrome secondary to pituitary adenomas in the medical literature. Before the acronym "SUNCT syndrome" had been proposed, a patient with a non-functioning pituitary macroadenoma and trigeminal neuralgia attacks, compatible with a diagnosis of SUNCT syndrome, had been described. ${ }^{12}$ The differential diagnosis between trigeminal neuralgia and SUNCT can be difficult. ${ }^{13-17}$

Two patients with SUNCT syndrome and prolactinomas have been described; one of the patients had a microprolactinoma while the other had a macroprolactinoma with cavernous sinus invasion. ${ }^{18}$ Recently, we have described a patient with SUNCT syndrome and microprolactinoma. ${ }^{19}$

The pathophysiology of pituitary-associated headache is poorly understood, although dural stretch, cavernous sinus invasion, and local pressure effects have been proposed as possible mechanisms. ${ }^{20-22}$ In all the case reports of SUNCT syndrome secondary to pituitary tumour, including the case described here, the SUNCT attacks occurred on the side ipsilateral to the side of the tumour, suggesting a role for a mechanical mode of action. However, though a mechanical effect may be a conceivable mechanism for the headache in cases of pituitary macroadenomas and cavernous sinus invasion, it is not a satisfactory explanation for microadenomas. Furthermore, a recent study in patients with pituitary tumours has demonstrated that there is a poor correlation between headache score and tumour size (hence dural stretch), cavernous sinus invasion and degree of carotid artery invasion. ${ }^{23}$ A plausible explanation is that the headaches are predominantly neurohumorally mediated rather than mediated by the size or invasiveness of the tumour. In this respect, it is interesting to note that in all case reports of SUNCT syndrome secondary to pituitary tumour, except the case reported here, dopamine agonists induced SUNCT attacks. This indicates a role for the dopamine axis in the aetiology of pituitary-associated headache and indicates that understanding the role of the hypothalamopituitary axis in the pathophysiology of these headaches may be very instructive.

This case report highlights the importance of appreciating that SUNCT syndrome can occur secondary to a pituitary tumour. The significance of this is further underlined by the findings that headache was the initial presentation in all cases reported hitherto, and that there can be a considerable time lag before the onset of pituitary-related symptoms. In the case reported by Ferrari et al, there were no pituitary symptoms despite a 10-year history of headaches. ${ }^{12}$ In the two cases reported by Massiou et al, the headaches preceded the pituitary symptoms by 3 and 4 years respectively. ${ }^{18}$ All patients presenting with suspected SUNCT syndrome should be questioned for symptoms associated with pituitary neoplasms, either due to hormonal imbalance or mass effect. Both the pituitary and posterior fossa abnormalities emphasise the absolute need for a cranial MRI, including an adequate view of the pituitary. In addition, these patients should have a screen for basal hormone measurements, including prolactin, TSH, free thyroxine, cortisol, ACTH, LH, FSH, oestrogen, testosterone and GH.

\section{ACKNOWLEDGEMENT}

PJG is a Wellcome Trust Senior Research Fellow.

\author{
Authors' affiliations \\ M S Matharu, M J Levy, P J Goadsby, Headache Group, Institute of \\ Neurology \\ R T Merry, The National Hospital for Neurology and Neurosurgery, \\ Queen Square, London, UK \\ Competing interest: None declared
}

Correspondence to: Professor P J Goadsby, Institute of Neurology, Queen Square, London WCIN 3BG UK; peterg@ion.ucl.ac.uk 


\section{REFERENCES}

1 Sjaastad O, Russell D, Horven I, et al. Multiple neuralgiform unilateral headache attacks associated with conjunctival injection and appearing in clusters. A nosological problem. Proceedings of the Scandinavian Migraine Society. Arhus 1978:31.

2 Pareja JA, Sjaastad O. SUNCT syndrome. A clinical review. Headache 1997;37:195-202.

3 May A, Bahra A, Buchel C, et al. Functional MRI in spontaneous attacks of SUNCT: short-lasting neuralgiform headache with conjunctival injection and tearing. Ann Neurol 1999;46:791-73.

4 Goadsby PJ, Lipton RB. A review of paroxysmal hemicranias, SUNCT syndrome and other short-lasting headaches with autonomic features, including new cases. Brain 1997;120:193-209.

5 Headache Classification Committee of the International Headache Society Classification and diagnostic criteria for headache disorders, cranial neuralgias and facial pain, 2nd ed. 2002. http://www.i-h-s.org.

6 Bussone G, Leone M, Volta GD, et al. Short-lasting unilateral neuralgiform headache attacks with tearing and conjunctival injection: the first symptomatic case. Cephalalgia 1991;11:123-7.

7 Morales F, Mostacero E, Marta J, et al. Vascular malformation of the cerebellopontine angle associated with SUNCT syndrome. Cephalalgia 1994:14:301-2.

8 De Benedittis G. SUNCT syndrome associated with cavernous angioma of the brain stem. Cephalalgia 1996;16:503-6.

9 ter Berg HWM, Goadsby PJ. Significance of atypical presentation of symptomatic SUNCT: a case report. J Neurol Neurosurg Psychiatry 2001;70:244-6.

10 Moris G, Ribacoba R, Solar DN, et al. SUNCT syndrome and seborrheic dermatitis associated with craneosynostosis. Cephalalgia 2001;21:157-9.

11 Penart A, Firth M, Bowen JRC. Short-lasting unilateral neuralgiform headache with conjunctival injection and tearing (SUNCT) following presumed dorsolateral brainstem infarction. Cephalalgia 2001;21:

\section{6-9.}

12 Ferrari MD, Haan J, van Seters AP. Bromocriptine-induced trigeminal neuralgia attacks in a patient with pituitary tumor. Neurology 1988; 38:1482-4.

13 Sjaastad O, Kruszewski P. Trigeminal neuralgia and "SUNCT" syndrome: similarities and differences in the clinical picture. An overview. Funct Neurol 1992;7:103-7.

14 Goadsby PJ, Matharu MS, Boes CJ. SUNCT syndrome or trigeminal neuralgia with lacrimation. Cephalalgia 2001;21:82-3.

15 Sesso RM. SUNCT syndrome or trigeminal neuralgia with lacrimation and conjunctival injection? Cephalalgia 2001;21:151-13.

16 Benoliel R, Sharav Y. Trigeminal neuralgia with lacrimation or SUNCT syndrome? Cephalalgia 1998; 18:85-90.

17 Bouhassira D, Attal N, Esteve M, et al. SUNCT syndrome. A case of transformation from trigeminal neuralgia. Cephalalgia 1994; 14:168-70.

18 Massiou H, Launay JM, Levy C, et al. SUNCT syndrome in two patients with prolactinomas and bromocriptine-induced attacks. Neurology 2002;58: 1698-9.

19 Levy MJ, Matharu MS, Goadsby PJ. Dopamine-agonist induced headache with prolactinoma: two cases and a review of the literature. Eur J Neurol 2003:10:169-73.

20 Abe T, Matsumoto K, Kuwazawa J, et al. Headache associated with pituitary adenomas. Headache 1998;38:782-6.

21 Powell M, Stafford L. The management of pituitary tumours: a handbook. London: Churchill Livingstone, 1996.

22 Arafah BM, Prunty D, Ybarra J, et al. The dominant role of increased intrasellar pressure in the pathogenesis of hypopituitarism, hyperprolactinemia, and headaches in patients with pituitary adenomas. $J$ Endocrinol Metab 2000;85:1789-93.

23 Levy MJ, Jager HR, Matharu MS, et al. Pituitary tumours and headache: does size matter? Cephalalgia 2002;22:592.

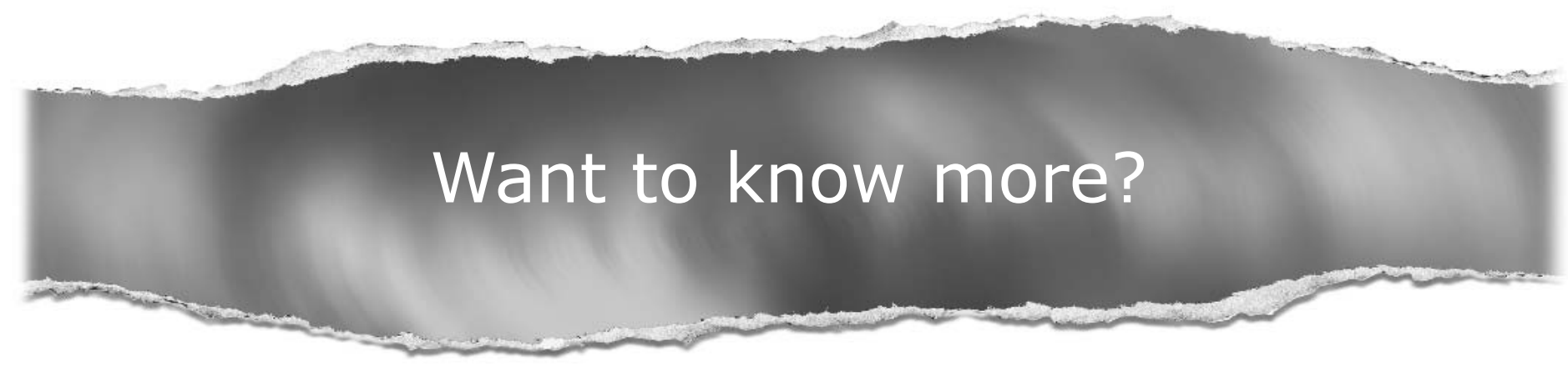

\section{Data supplements}

Limited space in printed journals means that interesting data and other material are often edited out of articles; however, limitless cyberspace means that we can include this information online.

Look out for additional tables, references, illustrations.

\section{www.jnnp.com}

